\title{
Basic Holographic Characteristics of a Panchromatic Light Sensitive Material for Reflective Autostereoscopic 3D Display
}

\author{
Ts. Petrova, B. Ivanov, K. Zdravkov, D. Nazarova, E. Stoykova, G. Minchev, and V. Sainov \\ Central Laboratory of Optical Storage and Processing of Information, Bulgarian Academy of Sciences, P.O. Box 95, Sofia 1113, Bulgaria
}

Correspondence should be addressed to V. Sainov, vsainov@optics.bas.bg

Received 3 October 2007; Accepted 26 March 2008

Recommended by John Watson

\begin{abstract}
Basic holographic characteristics of a newly developed panchromatic ultrafine grain silver halide light sensitive material for RGB recording of reflective holographic screen for autostereoscopic 3D display are presented. The average grain size is less than $10 \mathrm{~nm}$ which ensures high resolution, diffraction efficiency, and signal-to-noise ratio (more than $100: 1$ ) in a large dynamic range for RGB reflective holographic recording. The decrease of the diffraction efficiency in recording of scattering objects is less than $30 \%$ from the maximal values for specular reflection. The analysis of color recording of the reflective holographic screen with one viewing zone is presented on the basis of the so-called "sandwich" structure built of two layers for multiple holographic recording in blue, green, and red spectral regions.
\end{abstract}

Copyright (C) 2009 Ts. Petrova et al. This is an open access article distributed under the Creative Commons Attribution License, which permits unrestricted use, distribution, and reproduction in any medium, provided the original work is properly cited.

\section{Introduction}

Although the convergence conflict continues to be a serious problem of auto stereoscopic displays, the recent years marked a substantial progress in their development. More sophisticated and improved screens as a lenticular lens sheet, a convex lens array, and a holographic screen are under investigation [1-4]. The inherent property of a holographic screen for autostereoscopic imaging makes possible design of high-resolution "hybrid" systems and combination of different diffractive optical elements in a large image display with multiple high-quality viewing zones. Color holographic imaging is unquestionably the most perfect imaging technology since the reconstructed images are almost indistinguishable from the original scene. For example, a 40inch hybrid hologram screen is reported, which combines a Fresnel lens with a volume transmission RGB hologram [2]. In the described system, the 2D images from the "left" and "right" projectors form simultaneously the virtual images of the ground glass reconstructed from a hologram, which are converted by the Frenel lens to the real images in the viewing zone. The influence of color dispersion and chromatic aberrations, which are typical for transmission holograms in reconstruction with incoherent white light, is decreased by a proper choice of conditions for holographic recording of the ground glass.

A superior solution is to use a volume hologram of a reflection (Denisyuk's) type due to the absence of distortions and chromatic aberrations in white light "point source" reconstruction and better wavelength selectivity. The advantage of a reflection hologram is its filtering property which, due to Bragg diffraction, ensures selection of those wavelengths from the white light spectrum that have been used for recording. This unique property makes the reflection hologram a valuable holographic optical element (HOE) for design of reflective holographic screens with one or multiple viewing zones for "left" and "right" images in an autostereoscopic display. Projection in succession of 2D pictures onto the holographic screen reconstructs dynamic 3D images. For example, such a screen for a holographic movie has been reported 30 years ago by Serov and Komar [5]. A more sophisticated holographic reflective screen can be realized on the basis of recently advanced digital micromirror devices (DMDs) by implementing the idea of M.S. Ivanov to arrange a large number of micromirrors in a screen for creation of multiple viewing zones [5]. Reflective HOEs can be used also as screen and color filters for reflective liquid crystal displays. This idea is realized in [6], where 
an improved quality of images is achieved with holographic recording on DuPont photopolymers. Photopolymers, as typical phase-modulated media, exhibit very high diffraction efficiency at the expense of lower sensitivity in the red spectral region and limited dynamic range.

Properties of the holographic light sensitive materials are crucial for recording high-quality full-color holograms or HOEs. The most appropriate candidate for creation of a large size reflective holographic display is the silver halide ultrafine grain material due to its high diffraction efficiency, resolution, signal-to-noise ratio and sensitivity in the visual spectral range. The silver halide materials outperform the photopolymers in sensitivity in the visible and in the dynamic range. They have, however, lower diffraction efficiency than the photopolymers and suffer from low signalto-noise ratio due to increased light scattering in the blue spectral region. Increase of diffraction efficiency is achieved by bleaching of the silver halide holograms. The scattering problem can be solved by creation of a nanoparticle (5$10 \mathrm{~nm}$ ) emulsion.

Although the photographic silver halide emulsions have more than a century history of development and application, currently there is a lack of commercially-available suitable materials for multicolor holographic recording. At the moment, due to invasion of digital photosensors in photographic industry, production of silver halide light sensitive materials undergoes substantial reduction. Many of former producers of such materials as Agfa, Kodak, and other firms have entirely stopped industrial production. Today, the materials produced by Slavich are the only available on the market. Limited quantities of silver halide emulsion are produced in research laboratories, but mainly for scientific applications. Development of a new nanoparticle, high sensitivity $\left(<2 \mathrm{~mJ} \mathrm{~cm}^{-2}\right)$ low light-scattering, panchromatic silver halide emulsion as a commercial product will have many spheres of impact as security, cultural heritage and modern art, advertising and display systems, including future $3 \mathrm{D}$ dynamic holographic display (3D-TV) $[7,8]$.

The aim of this work is to present the recently obtained results in development of an ultrafine grain panchromatic silver halide emulsion for high-quality recording of RGBreflection holograms for the needs of autostereoscopic video display. The average grain size in the emulsion is less than $10 \mathrm{~nm}$ which ensures its high resolution, diffraction efficiency, and signal-to-noise ratio (more than $100: 1$ ) in a large dynamic range for RGBreflective holographic recording. Some promising preliminary research connected with the temporal stability of the emulsion has been reported in [9]. In this paper, we report basic holographic characteristics of the emulsion and we analyze color recording of the reflective holographic screen with one viewing zone on the basis of the so-called "sandwich" structure $[10,11]$ which is built of two layers for multiple holographic recording in blue, green, and red spectral regions.

\section{Exposure and Spectral Characteristics}

On the basis of experience gained in materials for monochrome recording, we have developed a panchromatic

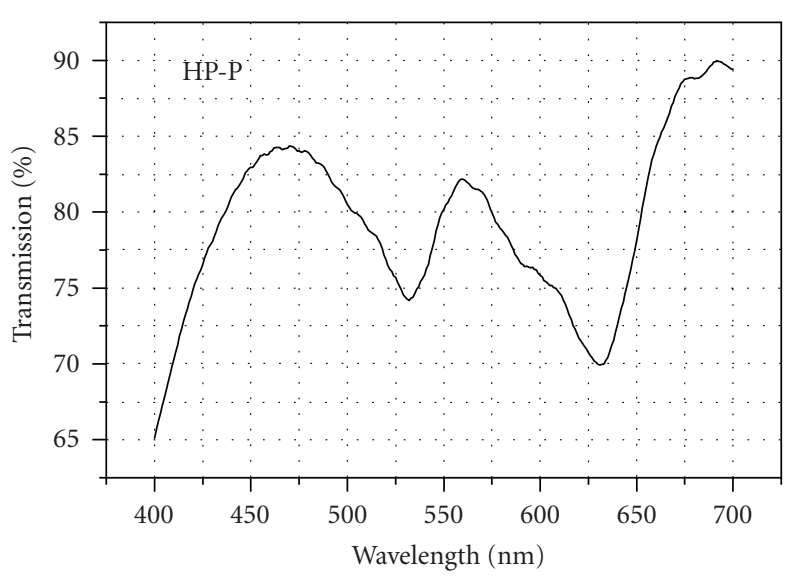

Figure 1: Transmission spectra of panchromatic silver halide light sensitive plates HP-P.

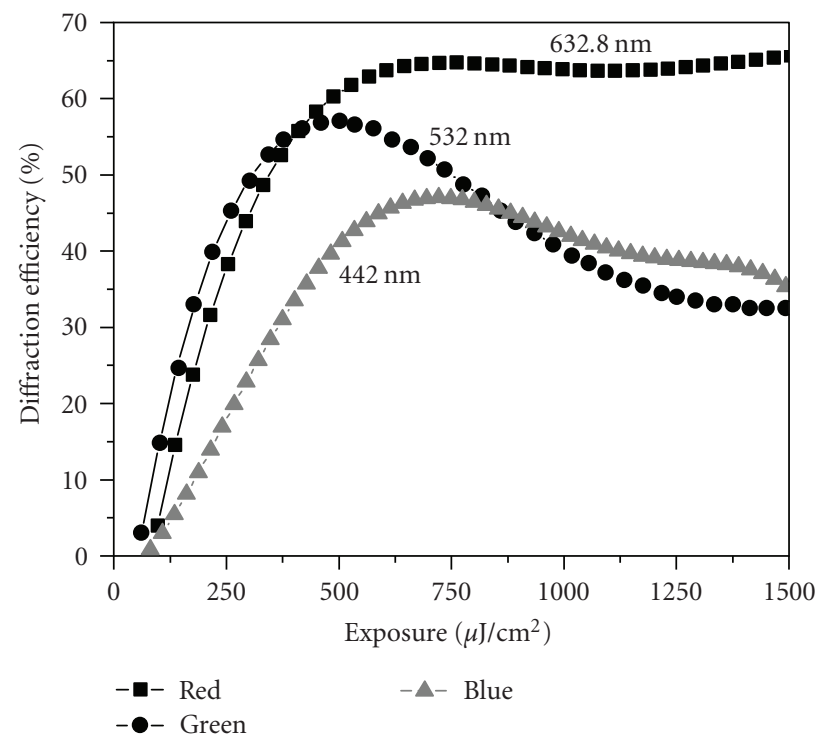

FIGURE 2: Exposure characteristics of HP-P for reflection RGB holographic recording.

silver halide emulsion for recording in blue, green, and red spectral regions. Silver halide recording materials are typical representatives of the so-called discrete recording media since the process of recording occurs in isolated particles which are suspended in the carrying matrix (gelatin). Spatial distribution of silver halide grains after developing and processing corresponds to intensity distribution in the recorded interference pattern. To ensure purely phase recording with high diffraction efficiency (almost 100\%), the developed silver grains are transformed into transparent particles using the so-called "bleaching." Thus light undergoes phase modulation due to the different refractive indices of the bleached grains and the carrying matrix. The spatial resolution depends on the size of the initial grains. More specifically, for low noise holographic recording, especially at short wavelengths, their size should be less than $10 \mathrm{~nm}$. Technologically, producing of such materials is a rather 
complicated task because of the thermodynamic instability of the grains and photosensitizing. The thermodynamic instability leads to growth of the grains and deterioration of the holographic characteristics as sensitivity, diffraction efficiency, and signal-to-noise ratio. It is well known that the temporal stability of the emulsion strongly correlates with its monodispersity. Another factor that substantially affects the lifetime of the silver-halide holographic materials is the temporal stability of the used photosensitizers for recording in the green and red spectral regions. For recording in blue (at wavelengths less than $450 \mathrm{~nm}$ ) spectral region a natural sensitivity of silver-halide materials is usually used.

The basic parameters of the developed emulsion as average grain size, polydispersity, and temporal stability were measured under laboratory conditions using preliminary calibrated nephelometric and refractometric techniques at $35^{\circ} \mathrm{C}$. The average grain size was determined by differential measurement of light scattering at $434 \mathrm{~nm}$. Evaluation of the light scattering dependence on the grain size was made by using transmission electron microscopy [12]. The polydispersity was estimated by using the nephelometer multiangle BI-200SM for the diluted in distilled water emulsion $(1.2: 24)$. We obtained that the average size of the silver halide grains in the synthesized emulsion was less than $10 \mathrm{~nm}$. Preparation of the emulsion was based on the wellknown "double jet" technique, but without using "freezing and thawing" like in Slavich materials PFG-01-PFG-03C, proposed by Kirillov [13, 14]. The developed emulsion was coated onto a glass substrate forming a light-sensitive layer with thickness of eight micrometers.

The transmission spectrum of the obtained panchromatic holographic plates (denoted below as HP-P), measured by Carry 5E spectrophotometer, is shown in Figure 1 [9]. To measure the diffraction efficiency of HP-P, we recorded reflection holograms of two collimated beams under CW laser irradiation at three different wavelengths $-442 \mathrm{~nm}$ (He-Cd laser), $532 \mathrm{~nm}$ (frequency doubled diode pumped solid state laser-DDPSS), and $632.8 \mathrm{~nm}$ (He-Ne laser). Developing was made with the well-known SM-6 developer with the following composition: ascorbic acid: $18 \mathrm{~g}$, sodium hydroxide: $12 \mathrm{~g}$, phenidone: $6 \mathrm{~g}$, sodium phosphate dibasic: $28.4 \mathrm{~g}$, water: 11 . The amplitude holograms were transformed into phase holograms by bleaching with the PBU-Amidol bleacher (Slavich) with composition: potassium persulphate: $10.0 \mathrm{~g}$, citric acid: $50.0 \mathrm{~g}$, cupric bromide: $1.0 \mathrm{~g}$, potassium bromide: $20.0 \mathrm{~g}$, amidol: $1.0 \mathrm{~g}$, water-to 1.01 [6]. To compensate the shrinkage of the layers after chemical processing and to ensure reconstruction of the Bragg reflection holograms at the wavelengths of recording, the suitable swelling was performed before drying in a bath of $5 \%$ water solution of collagen hydrolizate for 5 minutes at $20^{\circ} \mathrm{C}[15]$.

The exposure characteristics measured at the recording wavelengths are shown in Figure 2. As it can be seen, the dynamic range (linear part of the exposure characteristic) is $0.05-0.6 \mathrm{~mJ} / \mathrm{cm}^{2}$ for recording in the blue $(442 \mathrm{~nm}), 0.05-$ $0.5 \mathrm{~mJ} / \mathrm{cm}^{2}$ for recording in the green $(532 \mathrm{~nm})$, and $0.05-$ $0.75 \mathrm{~mJ} / \mathrm{cm}^{2}$ for recording in the red $(632.8 \mathrm{~nm})$ spectral regions. The spectral dependences of the diffraction effi-

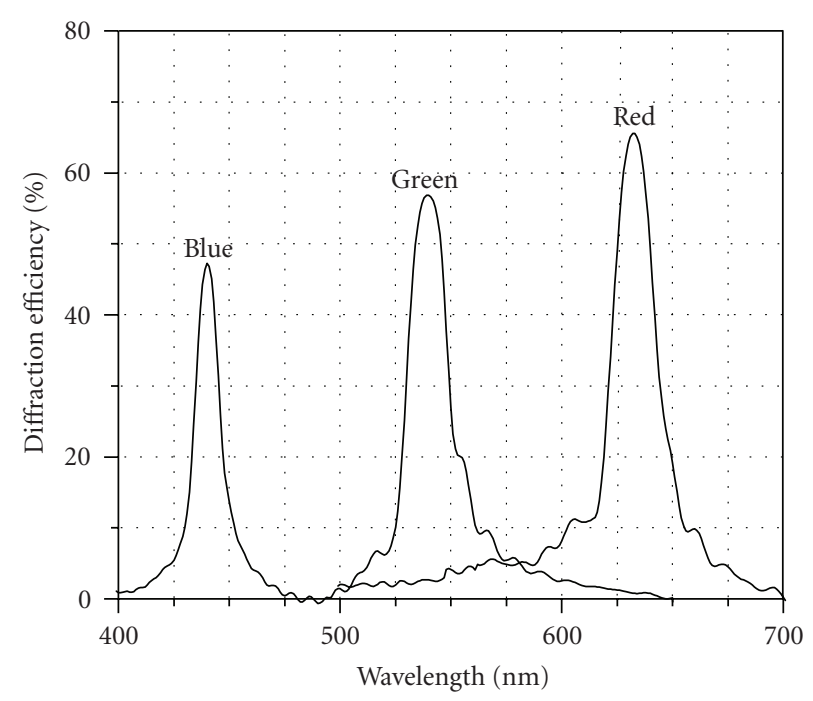

FIGURE 3: Spectral dependence of diffraction efficiency of bleached reflection holograms of two collimated beams for a single exposure in the blue $(442 \mathrm{~nm})$, green $(532 \mathrm{~nm})$, and red $(632.8 \mathrm{~nm})$ spectral regions.

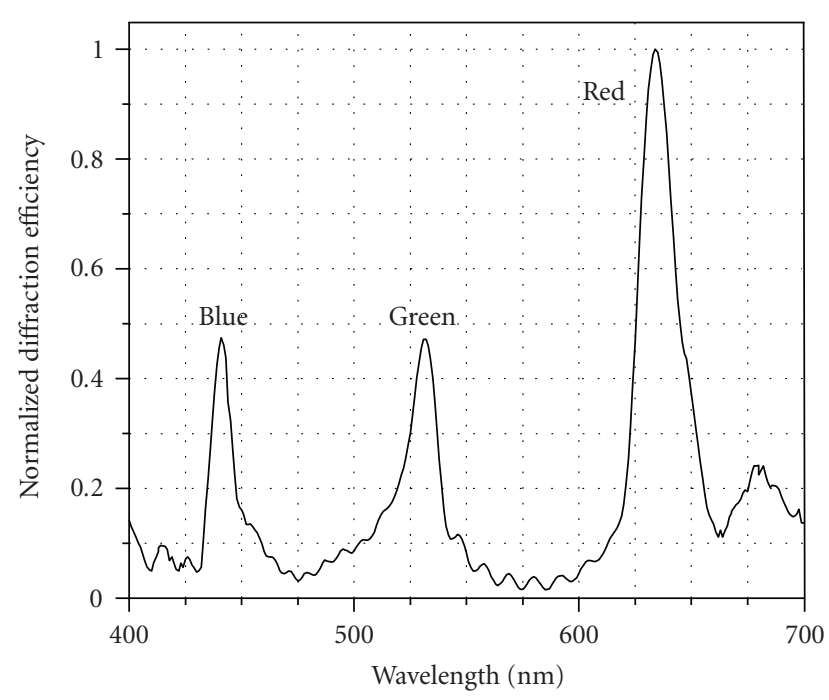

Figure 4: Spectral dependence of diffraction efficiency of bleached reflection holograms for multiple RGB recording of collimated beams in visible spectral range.

ciency, $\eta$, in the case of a single exposure made at each recording wavelength are given in Figure 3 . We see that we can expect values above $40 \%$ in the blue, $50 \%$ in the green, and $60 \%$ in the red for reconstruction with the used recording wavelengths. As the dynamic range of the refractive index modulation for all bleached silver halide materials is limited, being typically less than 0.08 for BBVPan plates [16] and less than 0.05 for the HP-P, in the case of multiplexing RGBholographic recording onto a single plate the diffraction efficiency of the individual holograms diminishes by a factor equal to the number of recordings in power 1.5-2. This is clearly seen in Figure 4, which 


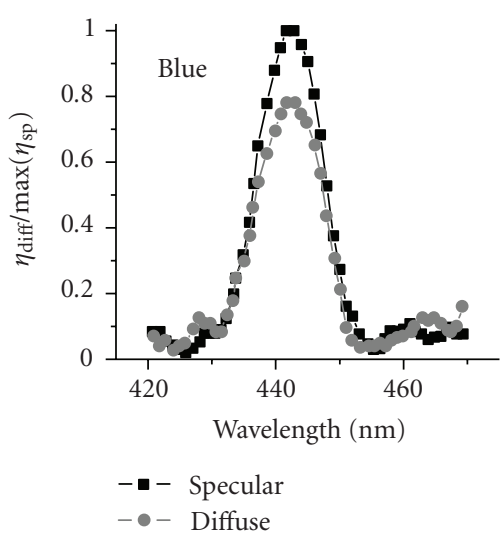

(a)

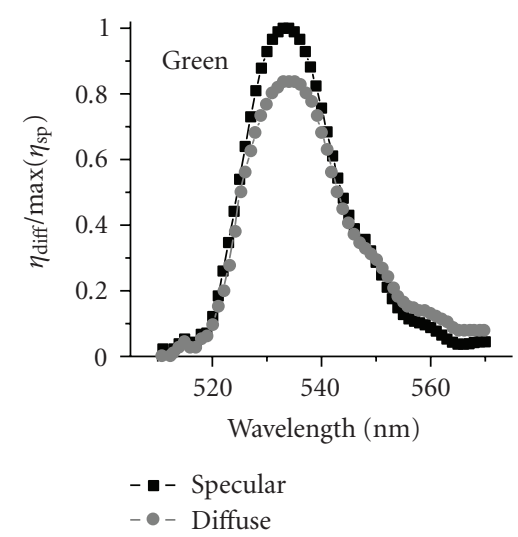

(b)

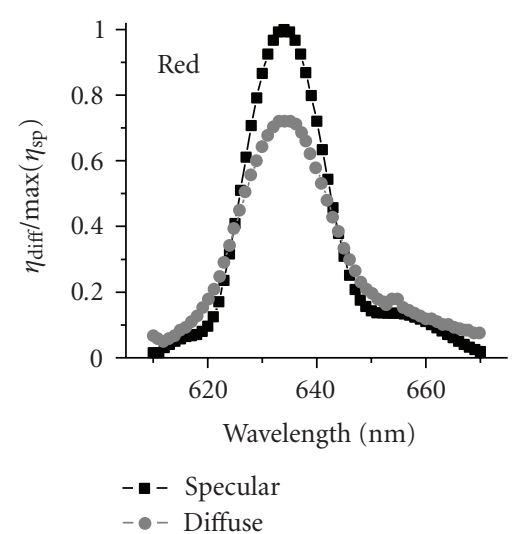

(c)

FIGURE 5: Spectral dependence of diffraction efficiency in recording of specular $\left(\eta_{\text {sp }}\right)$ and diffusely reflected objects $\left(\eta_{\text {diff }}\right)$ in red, green, and blue spectral regions.

depicts the spectral dependence of diffraction efficiency of a multiplexed reflection hologram recorded with the three wavelengths. The decrease of the efficiency in recording of light scattering objects is not more than $20 \%$ for the all used wavelengths, as is shown in Figure 5. The full width at half maximum (FWHM) of the curves for the diffuse reflection is practically the same as for specular reflection which is due to the high signal-to-noise ratio of recording (more than $100: 1)$. The obtained result is especially important for recording in the blue region. As a whole, the measured characteristics of the HP-P are promising for RGBrecording of reflection holograms and for correct color balance in reconstruction with incoherent "point source" white light.

The developed panchromatic ultrafine grain silver halide material HP-P has been successfully used for recording of Denisyuk's color reflection holograms by $\mathrm{CW}$ and pulse (30-40 nanoseconds) generating lasers in the spectral range $440 \mathrm{~nm}-660 \mathrm{~nm}$, as well as for recording of monochrome holograms by temperature stabilized diode lasers at $636 \mathrm{~nm}$, $658 \mathrm{~nm}$, and $672 \mathrm{~nm}$.

\section{Reflective Holographic Screen for Autostereoscopic 3D Display}

The optical arrangement for recording of an RGBreflective holographic screen with one viewing zone for autostereoscopic display is presented in Figure 6. The recording setup

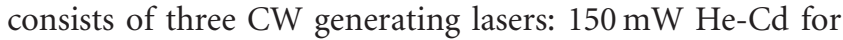
blue ( $422 \mathrm{~nm}), 2 \mathrm{~W}$ DDPSS double-frequency laser for green $(532 \mathrm{~nm})$, and $70 \mathrm{~mW} \mathrm{He}-\mathrm{Ne}$ for red $(632.8 \mathrm{~nm})$ spectral regions. Removable beam splitters (RBs) and mirrors (RMs) are adjusted before every individual recording in order to use the same beam expanders (B) and spatial filters (S) for each consecutive exposure.

Six individual holograms are required for creation of a single viewing zone of the 3D display for the "left" and "right" directions of observation. Recording of individual holograms in succession on a single plate by multiple exposures causes substantial decrease of diffraction efficiency. An

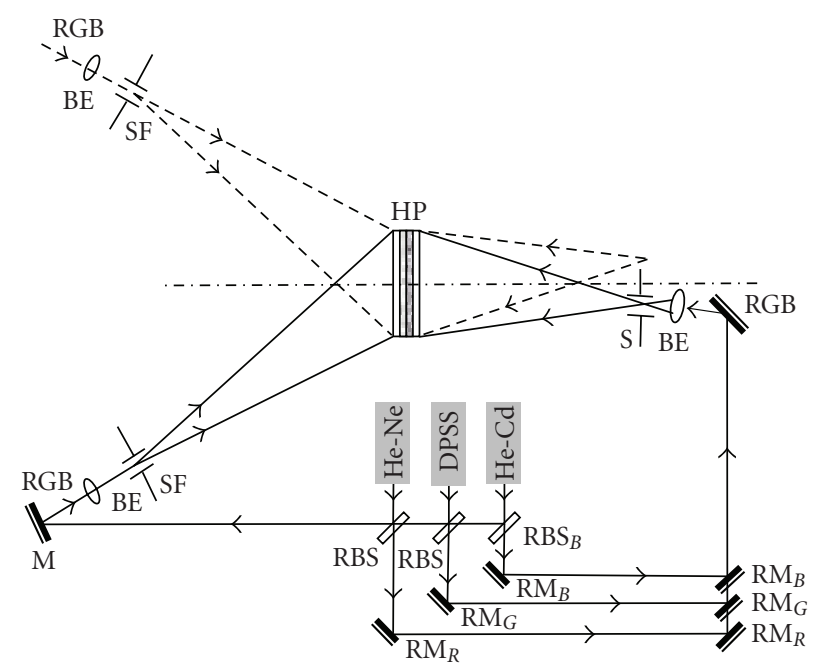

FIGURE 6: Optical arrangement for recording of reflective holographic screen.

effective way to solve the problem is to use the so-called "sandwich" structure $[10,11]$ which may consist of two or three light sensitive layers for separate hologram recording in blue, green, and red spectral regions. In the "sandwich" structure formed by two silver halide plates, the one plate is used for recording in the blue and green spectral regions while the other-for recording in the red. Assembling of the plates for reconstruction is so chosen that the plate recorded in the blue and green light is first on the way of the reconstructing beam and the plate recorded in the red is put behind.

Successful RGBreconstruction depends on correct energy balance of light exposures within the dynamic range of the recording material for different wavelengths. The total diffraction efficiency, $\eta_{\Sigma}$, in the case of a "sandwich" structure is determined from

$$
\eta_{\Sigma}=\frac{I_{d B}}{I_{0 B}}+\frac{I_{d G}}{I_{0 G}}+\tau^{4} \frac{I_{d R}}{I_{0 R}}=\eta_{B}+\eta_{G}+\tau^{4} \eta_{R},
$$




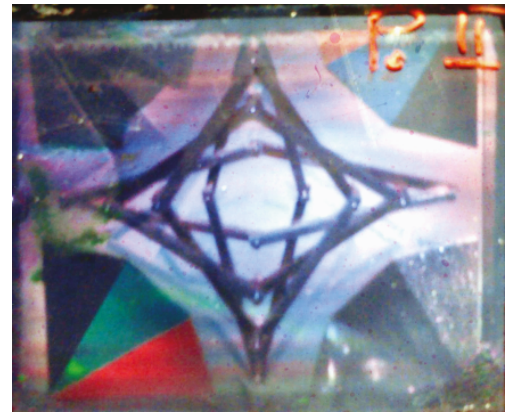

(a)

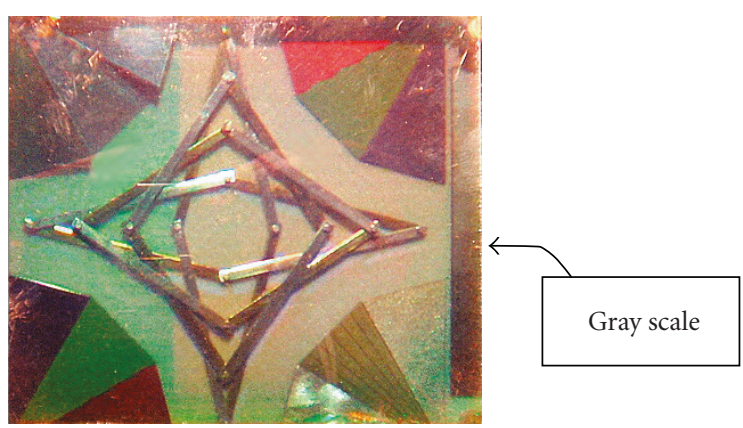

(b)

FIGURE 7: Reconstructed images of a test object from a single layer (a) and two layers (b) reflection holograms recorded on ultrafine grain light sensitive material HP-P.

where $I_{d B}, I_{d G}, I_{d R}$ and $I_{0 B}, I_{0 G}, I_{0 R}$ are the intensities of the diffracted and reconstructing light in the blue, green, and red spectral regions, respectively, $\tau$ is the amplitude transmittance of the developed holograms in the red spectral region. If for simplicity, we accept that

$$
I_{0 B}=I_{0 G}=I_{0 R}=I_{0},
$$

the total diffraction efficiency becomes

$$
\eta_{\Sigma}=\frac{I_{d B}+I_{d G}+\tau^{4} I_{d R}}{I_{0}}
$$

Diffraction efficiency of a bleached HP-P hologram diminishes approximately by a factor $(1 / 2) K^{2}$ at multiexposure recording, where $K$ is the number of exposures. Therefore, diffraction efficiencies of the individual holographic gratings in the sandwich structure that have been recorded with blue, green, and red light, respectively, for the left and right directions of the reference beam (see Figure 6) decrease with the number of exposures as

$$
\eta_{B}=\frac{\eta_{B \max }}{8}, \quad \eta_{G}=\frac{\eta_{G \max }}{8}, \quad \eta_{R}=\frac{\eta_{R \max }}{2},
$$

where $\eta_{B \max }, \eta_{G \max }, \eta_{R \max }$ are the maximum values of diffraction efficiencies at single recording in the blue, green, and red parts of the spectrum. Finally, for the total diffraction efficiency, we obtain

$$
\eta_{\Sigma}=\frac{\eta_{B \max }+\eta_{G \max }}{8}+\tau^{4} \frac{\eta_{R \max }}{2} .
$$

If we suppose that $\eta_{B \max }=\eta_{G \max }=\eta_{R \max }=\eta_{\max }$ and $\tau \sim 1$, the efficiency in the case of a sandwich hologram will be $(3 / 4) \eta_{\max }$, whereas for a single plate with $K=6$ exposures it is equal to $(1 / 18) \eta_{\max }$, that is, we have almost 14 times better optical response of the sandwich structure. Improvement of quality of the reconstructed image in the case of a two-layer reflection hologram is clearly seen in Figure 7 . The figure presents reconstruction of a test object which is build of areas with different color, texture, and reflective properties. The most convincing proof of better signal-to-noise ratio in the case of the sandwich hologram is quality of reconstruction of the gray scale incorporated in the scene.
In accordance with the color diagram in Figure 8, realistic reconstruction of a white color for the used wavelengths is achieved if the ratio between the intensities of reconstructed waves is

$$
I_{d B}: I_{d G}: \tau_{d R}^{4} I_{d R}=1: 0.68: 0.95
$$

Having in mind the experimentally obtained (Figure 3 ) maximum values of diffraction efficiency $\eta_{B \max }=0.48$; $\eta_{G \max }=0.57 ; \eta_{R \max }=0.66$, respectively, and taking $\tau \sim 0.85$, we obtain from (1) that the ratio between the intensities of the light waves for reconstruction of the primary colors should be

$$
I_{0 B}: I_{0 G}: I_{0 R}=0.44: 0.25: 0.15 \text {. }
$$

Obviously, some other recording combinations with corresponding ratio of light exposures can be proposed to realize effective mixing of holograms in a "sandwich" structure.

In general, the total exposure $E_{T}$ delivered to a single layer can be described as

$$
E_{T}=\sum_{i=1}^{K}\left[\left(E_{\max }^{C_{i}}-E_{\min }^{C_{i}}\right) \times\left(\alpha_{i}\right)\right]+E_{\min }^{C_{1}},
$$

where $K$ is the number of recordings onto the light sensitive material, $E_{\min }^{C_{i}}$ and $E_{\max }^{C_{i}}$ are minimal and maximal exposures, respectively, in the linear part of exposure characteristic for the monochrome recording at the corresponding wavelength which is indicated with a special color pointer $C_{i}$. For example, in the case of recording of four diffraction gratings with blue and green light at the angles chosen for the left and right directions (Figure 6) the color pointer indicates two times recording at $442 \mathrm{~nm}$ and two times recording at $532 \mathrm{~nm}$. The color filling coefficient $0<\alpha_{i} \leq 1$ is introduced to ensure optimal use of the dynamic range of the light sensitive material and to avoid its saturation. The proper choice of the color filling coefficient is essential for additive color mixing in reconstruction. The second term in (8) gives the necessary offset which is accumulated during the first exposure.

To convert the virtual to real image, the reconstruction is produced from the opposite directions to the recorded 


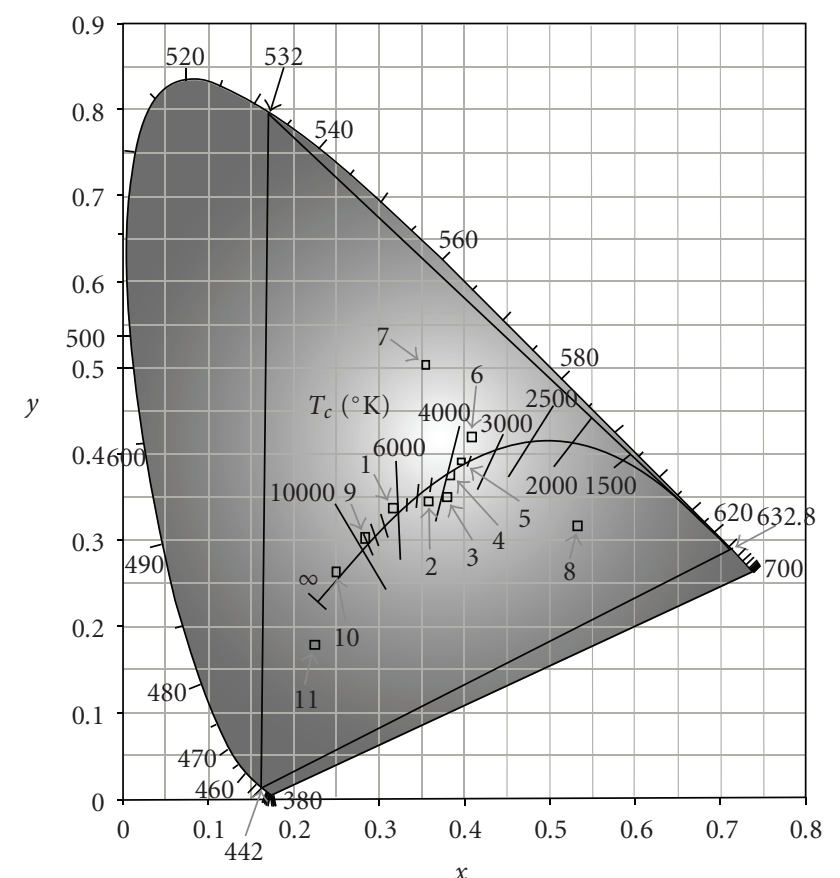

Figure 8: Diagram of color coordinates and color temperatures of real objects (1) white surface, (2) snow, (3) white human skin, (4) grey stone, (5) sand, (6) yellow flower, (7) green grass, (8) red flower, (9) blue sky, (10) lake on a sunny day, (11) blue flower.

screen, as shown in Figure 9. Liquid crystals or DMD projectors, available on the market, are appropriate for reconstruction of the images from the holographic screen, with possibility for additional corrections of the color balance. The size of the viewing zone depends on the size of the exit pupils of the projection objectives. The described approach could be applied for creation of multiview auto stereoscopic display-at least three viewing zones. The diffraction efficiency will drop considerably, but for the high-power projectors efficiency in the order of $1-2 \%$ is completely enough for creation of high-quality images if the requirement for a very high signal-to-noise ratio is satisfied, as is the case of HP-P (more than $100: 1$ ).

\section{Conclusion}

In summary, the basic holographic characteristics of bleached reflection holograms, recorded onto panchromatic silver halide light sensitive plates HP-P as exposure and spectral dependences of diffraction efficiency in recording of specular and diffusely reflected objects in the red, green, and blue spectral region, signal-to-noise ratio and the dynamic range, were investigated. The dynamic range is $0.05-0.6 \mathrm{~mJ} / \mathrm{cm}^{2}$ for recording in the blue $(442 \mathrm{~nm}), 0.05-$ $0.5 \mathrm{~mJ} / \mathrm{cm}^{2}$ for recording in the green $(532 \mathrm{~nm})$, and 0.05 $0.75 \mathrm{~mJ} / \mathrm{cm}^{2}$ for recording in the red $(632.8 \mathrm{~nm})$ spectral region. Signal-to-noise ratio is more than 100:1 with maximal value of efficiency $40 \%$ in the blue, $50 \%$ in the green, and $60 \%$ in the red spectral region.

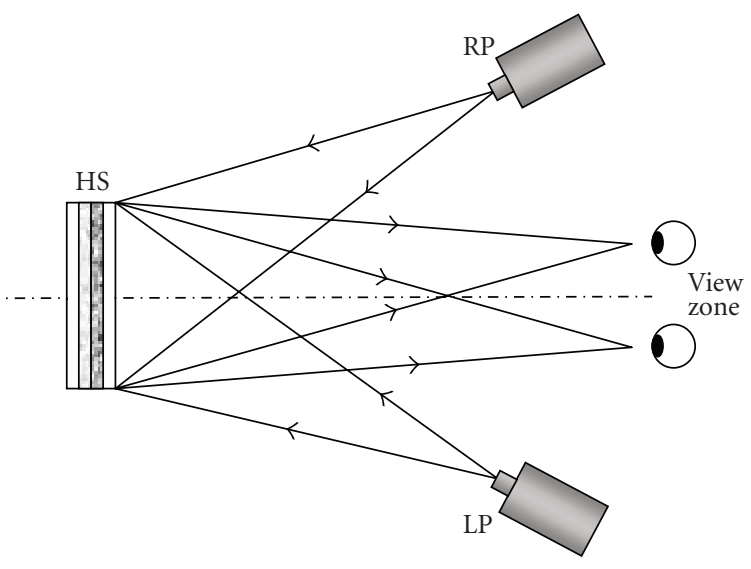

FIGURE 9: Optical arrangement for reconstruction of 3D images with one viewing zone from reflective holographic screen (HS) using left (LP) and right (RP) projectors.

The HP-P materials are the most promising candidates for creation of an RGBreflective holographic screen with a single and multiple viewing zones. Implementation of the socalled "sandwich" structure consisting of two light sensitive layers for separation of recordings in the red and in the green and blue regions, respectively, ensures acceptable diffraction efficiency for 3D autostereoscopic imaging for different viewers. It should be noted that, despite the technological difficulties connected with the wet chemical processing, the higher sensitivity of the silver halide materials makes them more suitable for large-size reflective screens than the photopolymers at comparable characteristics of the displayed images.

\section{Acknowledgment}

This work is supported by NoE EC Project 3DTV no. 511568 .

\section{References}

[1] H. Song, Y. Nakashima, Y. Momonoi, T. Honda, and T. Sina, "Wide viewing zone of auto stereoscopic 3-D display system by hybrid hologram screen," HODIC Circular, vol. 23, no. 3, pp. 6-9, 2003.

[2] H. H. Song, Y. Momonoi, T. Shibuya, and T. Honda, "Multi view 40-inch hybrid large hologram screen for auto stereoscopic 3-D display system," in Proceedings of the 3D Image Conference, pp. 1-4, Tokyo, Japan, July 2003.

[3] J. M. Kim, B. S. Choi, S. I. Kim, J. M. Kim, H. I. Bjelkhagen, and N. J. Phillips, "Holographic optical elements recorded in silver halide sensitized gelatin emulsions-part 1: transmission holographic optical elements," Applied Optics, vol. 40, no. 5, pp. 622-632, 2001.

[4] J. M. Kim, B. S. Choi, Y. S. Choi, J. M. Kim, H. I. Bjelkhagen, and N. J. Phillips, "Holographic optical elements recorded in silver halide sensitized gelatin emulsions-part 2: reflection holographic optical elements," Applied Optics, vol. 41, no. 8, pp. 1522-1533, 2002.

[5] V. Komar and O. Serov, Diplay Holography and Holographic Cinema, Izkustvo, Moscow, Russia, 1987. 
[6] Y. Higashikaki, T. Tokumaru, and K. Iwauchi, "Holographic directive reflectors for reflective color LCDs," http://sharpworld.com/corporate/info/rd/tj2/pdf/4.pdf.

[7] H. I. Bjelkhagen and D. Vukicevic, "Colour holography: a new technique for reproduction of paintings," in Practical Holography XVI and Holographic Materials VIII, vol. 4659 of Proceedings of SPIE, pp. 83-90, San Jose, Calif, USA, January 2002.

[8] H. M. Ozaktas and L. Onural, Eds., Three-Dimensional Television: Capture, Transmission, Display, Springer, Berlin, Germany, 2008.

[9] Ts. Petrova, N. Tomova, V. Dragostinova, S. Ossikovska, and V. Sainov, "Life-time of silver halide holographic materials comparative accelerating testing," in Holography 2005: International Conference on Holography, Optical Recording, and Processing of Information, vol. 6252 of Proceedings of SPIE, Varna, Bulgaria, June 2006.

[10] V. Sainov, G. Spassov, and S. Sainov, "Diffraction efficiency increasing in color display holograms recording," Journal of Scientific and Applied Photography and Cinematography, vol. 6, p. 413, 1981 (Russian).

[11] T. Kubota, "Recording of high quality color holograms," Applied Optics, vol. 25, no. 22, pp. 4141-4146, 1986.

[12] V. Sainov, M. Mazakova, and N. Koleva, "Characteristics of non-bleached reflection holograms," Comptes Rendus de l'Academie Bulgare des Sciences, vol. 34, no. 9, pp. 1241-1244, 1981.

[13] G. Lippmann, Compte Rendus de l'Académie des Sciences, vol. 112 , p. $274,1891$.

[14] N. I. Kirillov, The Basis for Developing Processes of Silver Halide Photo Materials, Izkustvo, Moscow, Russia, 1977.

[15] C. G. Stojanoff, H. Schuette, O. Brasseur, R. Kubitzek, and S. Tropartz, "Photochemical and thermal treatment of dichromated gelatin film for the manufacturing of holographic optical elements for operation in the IR," in Photopolymer Device Physics, Chemistry, and Applications II, vol. 1559 of Proceedings of SPIE, pp. 321-330, San Diego, Calif, USA, July 1991.

[16] H. Bjelkhagen, Silver Halide Recording Materials for Holography and Their Processing, Springer, Berlin, Germany, 1995. 